\title{
Some Significant Advances in Yarn Splicing Technology
}

\author{
N Gokarneshan*, K Velumani, Marudhachalam and Gokulaprabha \\ Department of Textile Technology, Park College of Engineering and Technology, India
}

Received: 眥December 15, 2017; Published: 眥January 24, 2018

*Corresponding author: N Gokarneshan, Department of Textile Technology, Park College of Engineering and Technology, Coimbatore, Tamil Nadu, India

\begin{abstract}
The article reviews some of the significant researches in the yarn splicing technology. The effect of important factors on the breaking force of wet spliced yarns has been studied by means of a central composite design, which is a statistical tool. The impact of vent pressure on the quality of yarn splicing when twisting and untwisting has been investigated. Modelling, analysis, and simulation of the air splice is done with the integration of two software's, so as to analyse the flow field and obtain the effect of speed and pressure of the inside the splicing chamber when changing the speed and the pressure while maintaining the diameter of the splicing, the vent hole diameter and the relative angle unaltered. The influence of cotton/polyester fibre blend ratios on spliced portion diameter at various splicing air pressures has been studied. Studies have been directed regarding the influence of various yarn splicing parameters on the strength, elongation and the appearance of spliced cotton/flax yarn. A number of independent variables have been considered. A half factorial experimental design has been used with two levels of each variable. The design and analysis of experiments have been achieved with a specific software.
\end{abstract}

Keywords: Blended yarn; Retained splice elongation; Retained splice strength; Pneumatic splicer system; Retained splice diameter; Software; Experimental design

\section{Introduction}

Yarn winding is the process of transferring yarn from bobbins to packages to facilitate in the subsequent processes of warping, weaving and knitting. In this process, both the yarn ends are joined together to provide the continuity of the yarn package and several yarn faults; neps, thin place, thick place are removed. Thus, more uniform yarn mass distribution and higher yarn evenness properties are obtained. The yarn ends are joined together by applying a blast of compressed air into a profiled device called a splicing chamber. The most important performance parameters of splicing are obtaining adequate strength and appearance at the splice point for all processing requirements. The diameter of spliced portion affects not only appearance of the splice joints but also physical characteristics such as packing density, strength, specific volume etc. Yarn diameter is an important property which affects the physical properties of the end products. Variations in yarn diameter cause undesirable effects such as imperfections in the knitted or woven fabric and difficulties in weaving preparation stages.
The achievement of an appropriate splice in winding is one of the most important factors for spinners to meet present requirements in terms of productivity and quality. Different splicing techniques, pneumatic, mechanical and electrostatic, were developed towards the end of the last century [1]. Over the last twenty years, many studies have tried to broaden the end use of pneumatic splicing, in the upward transformation process [2-4]. The mechanical properties of spliced yarns which affects the quality of the denim yarn are influenced by variations in parameters such as raw materials and process \& machine variables. Increasing some of these variable results can increase or decrease the performance of mechanical properties. Splicing is a technique which was especially developed to meet requirements in weaving where demands are severe. This is the manner of joining two ends after preparation by air under pressure $[5,6]$. The high-speed compressed air is used as the driving force of the air splice, after the process of yarn cutting, untwisting and twisting, to complete the yarn splicing, which is a typical application of fiber-media fluid dynamics in the textile industry [7]. 


\section{Central Composite Design to Determine Strength of Wet Spliced Post Sized Denim Yarns}

The abrasion, tensile strength \& elongation of the splices have been investigated $[8,9]$. It has been shown by linear regression and neural networks that the linear density of yarn is the most influential parameter on these physical properties. The various zones which participate in traction by defining the total mechanism of splices have been considered [10-12]. It has been shown especially that splice remains a failing technique in weaving, and it is essential to optimise it in order to improve certain input parameters. The technique of image analysis of morphology of splices has been used to prove that splicing is not reliable compared to the parent yarn [13]. In general, it is important that mechanical properties of wet spliced yarn after sizing should have the highest values. For this reason, the levels of the variables that affect the performance of the splice should be selected so that maximum properties are achieved.

The influence of four parameters has been investigated with the help of a central composite design for the experiments. Statistical analysis used for the results obtained from these experiments establishes a relationship of retained strength and retained elongation at break with these variables [14]. The details of the experiments and the subsequent analysis have been reported. Detailed regression analysis shows that while the factors of yarn count, Yc, length of splice, SL and quadratic effect, 2 SL are highly significant, their interactions with the other factors are not significant regarding the strength of spliced yarn. Although a sizable lack of fit sum of squares is present, the response surface equation describes the experimental system reasonably well for our purpose, since regression accounts for $80 \%$ and $83.4 \%$ of the total variation of the data. The model can thus be used to predict the response within reasonable limits. Sizing encourages the resistance of wet pneumatic-spliced denim yarns, and this was justified for the two recipes used. With this type of experimental design, we optimised the basis of input parameters and their impacts on the breaking strength of splice after sizing.

\section{Fluid Dynamics Software to Analyse Effect of Vent Pressure on Yarn Splicing}

Through the air-splicing process, the quality of the yarn all will be influenced by the speed and pressure of the compressed air, the volume and of the air injection, the length of the air injection time and etc. The air pressure should be appropriate, and be kept stable. When the compressed air gets consumed to a certain value, the air pressure increases accordingly and the fibers in the overlap portion will be intertwined better. However, when the air pressure exceeding the maximum critical pressure, the pressure promotes the fiber splicing, also has a negative impact on the yarn splicing. The reason is that excessively high compressed air will result in air disorders vortex, which affecting the fiber splicing and leading to a decline in the quality of splicing. It can be seen that a suitable air pressure for improving the splice quality is essential [15]. Due to the fact that it is difficult to directly observe the internal cavity of the air splice, and the mechanism of the splicing process is derived based on the experimental data.

The, the speed and the pressure of the air flow field in the internal splice were analyzed, with the help of Pro/E, ANSYS and other software. By changing the inlet pressure on the splice cavity, the diversification of the air flow field was studied, and the mechanism of the air splice was researched deeply, which provided a theoretical basis for the parameter optimization and structure design of the splicing chamber. Through the flow field analysis of the splice chamber, the velocity and the pressure distribution of the same cross-section at different inlet pressures were compared comprehensively and observed that the speed, the pressure and other parameters of the chamber increase with the growth of the inlet pressure. Combined the simulation analysis with the demand of practical work, it can be drawn that under the conditions of the splice chamber diameter of $15 \mathrm{~mm}$, the vent diameter of $5 \mathrm{~mm}$, the relative angle of $60^{\circ}$, and the vent pressure of $0.6 \mathrm{MPa}$, the effect of the splicing is best [16]. The analysis results have important significance to improve the effect of the yarn splicing and provide a theoretical basis for the design of the internal structure.

\section{Influence of Fibre Blending Ratios on Retained Splice Diameter}

In literature, some studies have been performed on the effect of splicing parameters such as splicing length, duration of splicing air blast, and splicing air pressure on strength and appearance of the spliced yarns [17-26]. Studies have been carried out on the effect of both fiber characteristics and splicing parameters on retained spliced diameter [27-29]. In these studies, yarn samples with different types of cotton fibers have been produced and applied splicing process. In literature, there is very limited study on effect of yarn blending ratio on spliced portion diameter at different slicing air pressures [30]. With the development in technology and improvement in human life standards, the consumer demands from a product increases day by day. In order to satisfy the requirements, the fiber blends are used with different ratios to make a full use of their specific characteristics. Each fiber has its own specific mechanical and chemical properties. The fiber characteristic properties such as length, bending, resilience, elasticity etc. determine the yarn properties. It is claimed that since the splicing process is based on the snarling of the fibers in yarn structure because of pressurized air, the splice joint diameter and spliced yarn strength will be affected by the fiber characteristics.

Attention has been directed towards study of the effect of fiber blend ratio on spliced portion diameter at different slicing air pressures. For this aim, three yarn samples $100 \%$ cotton, 80 20\% CO-PES and 50-50\% CO-PES were produced with 40/1 Ne. Each yarn sample is spliced at three different pressures; 4 bar, 5 bar and 6 bar [31]. The images of the yarn samples were acquired. The diameters of spliced portion and retained yarns are measured 
on the images of the spiced yarns. The effect of cotton-polyester fiber blending ratio on splice portion appearance performance was investigated. It can be said that the optimum splice pressure for the most appropriate splice appearance changes with respect to the fiber content of the yarn. For 4 and 5 bar splice air pressures, as polyester ratio of the yarn content was increased, the diameter of the splice portion also increased with respect to the diameter of parent yarn. For 6 bar air pressure, the splice joint diameter decreased with the increase in polyester content of the yarn samples. According to statistical analysis results, it can be revealed that the splice pressure and yarn fiber content have statistically significant effects on spliced yarn diameter. On the other hand, it can also be concluded that each pressure level has statistically different effects on spliced yarn diameter. With respect to the fiber blend ratio, 50-50\% CO-PES and 80-20\% CO-PES blend types have statistically similar effect on the diameter of spliced yarn. But $100 \%$ CO yarn statistically differs from $50-50 \%$ CO-PES and $80-20 \%$ COPES blend types.

\section{Effect of Splicing Factors on Some Properties of Cotton Flax Blended Yarn}

In the yarn winding process, ring yarn bobbins are converted into yarn packages which are more practical in the subsequent processes of warping, weaving, and knitting. This process not only ensures the continuity of the yarn by splicing yarn ends on different bobbins one by one, but also results in clearing of several yarn faults. Manual knotting has been a traditional practice for joining yarn ends together, but it can seriously deteriorate the resultant yarn and fabric appearance [32]. Pneumatic splicing is a relatively complex phenomenon as compared to manual knotting and is the most widely used technique for acceptable knot free joint. Previous studies revealed that a splice consists of three distinct zones, viz., twisting, wrapping and tucking or intermingling [33,34]. In a pneumatic splicing chamber, yarn ends to be spliced are overlapped, surplus yarn ends (as per specified splice length) are removed and a turbulent (Reynolds number up to 105) compressed air blast is released to perform the simultaneous action. Due to the complexity and instability of the splicing process, the process outputs are often unpredictable.

A couple of studies have been reported on how splicing and yarn parameters affect the strength, abrasion, bending and appearance of the spliced cotton and polyester/cotton blended yarns [35]. A statistical model has been developed to observe the effect of fibre friction, yarn twist and splicing air pressure on spliced cotton yarn performance [36]. Comparison of splicing performance of viscose staple fibre yarns made from ring, rotor, friction and air jet spinning technologies has been reported along with the splicing performance of synthetic filament yarns [37]. Some researchers have illustrated the effect of splicing parameters (splice length, duration of splice air blast and splice air pressure) on the tensile and structural characteristics of spliced yarn of different spinning technologies
[8]. They found that the difference in yarn structure, due to varied spinning technologies and yarn splicing parameters influence the performance of splice. Although the concept of pneumatic splicing is not new, there have so far been little published works available on the study of factors affecting the mechanical properties of spliced yarns, particularly no work has been reported on the cotton/flax blended yarns. Efforts have been directed towards the effect of different splicing parameters on strength, elongation and appearance of the spliced cotton/flax blended yarns (Figure 1).

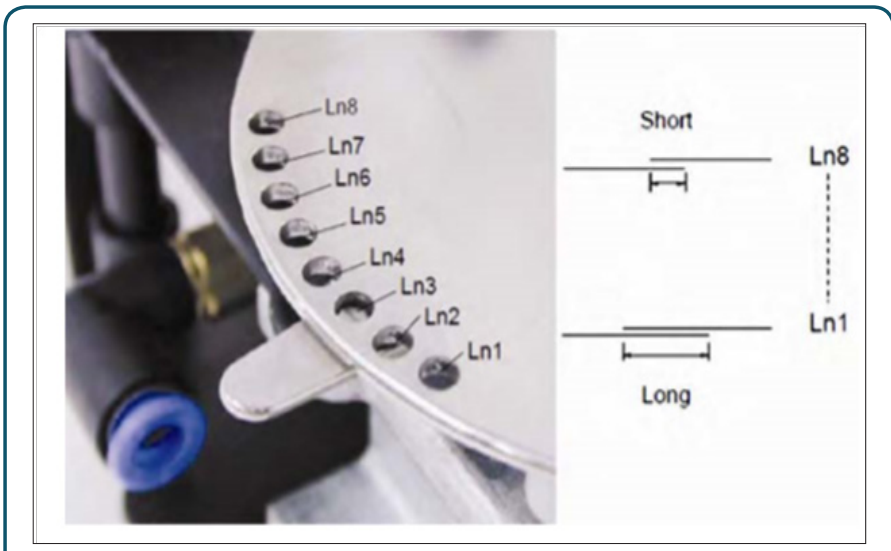

Figure 1: Splice length control lever [37].

The factors investigated are as follows:

a) Effect of yarn splicing parameters on retained splice strength

b) Effect of yarn splicing parameters on retained splice elongation

c) Effect of yarn splicing parameters on appearance of spliced yarn

Different splicing parameters affect the characteristics of spliced portion of yarn in different manner. Retained splice strength of yarn is mainly affected by the splicer nozzle type. Retained splice elongation is mainly affected by the type of untwisting pipe while the appearance of the spliced yarn is mainly affected by the splicer lever type. The $8 \mathrm{FB} 1^{\mathrm{A}}$ plate has better compatibility with HB lever and NOC untwisting plate, while FBOD (Appendix) plate has better compatibility with $\mathrm{H}$ lever with less twisting time give better retained splice strength. Similarly, the combination of less twisting time with shorter splice length and more twisting time with longer splice length give better retained splice elongation. Furthermore, the appearance of spliced portion of yarn was better if shorter twisting time is used for Ln1 position of control lever and longer twisting time was used for Ln3 position and control lever.

\section{Appendix}

A. It is the specification of the front plate on which the twisting nozzle is mounted in the splicing unit. It is used for proper guidance of untwisted yarn ends into the twisting nozzle. 
It has two profiles at upper and lower sides which are slightly offset relative to each other for proper guidance of yarn ends and holes for entry of yarn ends into untwisting pipes.

B. It is a type of yarn holding lever which holds the yarn ends after untwisting and keeps them in place during twisting in the untwisting nozzle. It has two arms for holding the upper and lower yarn ends during twisting/splicing.

C. It is a type of untwisting pipe. The untwisting performance can be controlled by changing the type of untwisting pipe as well as the position of a particular untwisting pipe in untwisting nozzle. This type of pipe is the standard untwisting pipe and can be used for coarse as well as fine counts. It has a length of $48 \mathrm{~mm}$ and diameter of $6 \mathrm{~mm}$. It is mainly used for untwisting of short fibres.

\section{It is a type /specification of front plate.}

\section{Conclusion}

The recent researches on yarn splicing technology have focussed on achieving optimum quality of yarn splice. One important aspect is the retained splice strength which in the case of wet spliced yarns can be controlled to some extent by suitable selection of certain factors related to the process of yarn splicing. A central composite design has been adopted so as to enable a better understanding of the manner in which the variables (yarn count, the duration of air and water joining) influence the breaking force of wet spliced yarns. Pneumatic splice makes use of high-speed compressed air as the driving force to complete the yarn splicing, noticing that the change of the vent pressure has a significant effect on yarn splicing. The impact of vent pressure on the quality of yarn splicing when twisting and untwisting has been investigated. Specific software has been used for modelling, analysis and simulation of the air splice. The findings of the analysis of the air splice will lay a solid foundation for the further optimized structure design.

The most important performance parameters of splicing are adequate strength and appearance at the splice point for all processing requirements. The diameter of spliced portion effects not only appearance of the splice joints but also physical characteristics such as packing density, strength, specific volume of the yarn. The influence of cotton/polyester fiber blend ratios on spliced portion diameter at various splicing air pressures has been studied. The diameters of spliced portion and retained yarns were measured by using Image program and the results were analyzed statistically. The influence of various yarn splicing parameters on the strength, elongation, and appearance of spliced cotton/flax yarn has been studied. Study of the various related parameters using factorial experimental design shows that the retained splice strength of yarn is mainly affected by the splicing nozzle profile. Retained splice elongation is mainly affected by the type of untwisting pipe while the appearance of the spliced yarn is mainly affected by the splicer lever type. Moreover, because of significant interactions, an optimal combination of splicing variables is required in order to achieve optimum retained splice yarn strength, elongation and appearance.

\section{References}

1. Bissman D (1981) Knotfree Yarns Joins by Splicing. International Textile Bulletin Spinning 3/81, 281.

2. Kaushik RCD, Sharma IC, Hari PK (1987) Effect of Fibre/Yarn Variables on Mechanical Properties of Spliced Yarn. Textile Research Journal 57(8): 490-494.

3. Kaushik RCD, Hari PK, Sharma IC, Sarkar AK (1987) Performance of Spliced Yarn in Warping and Weaving. Textile Research Journal 57(11): 670-673.

4. Kaushik RCD, Hari PK, Sharma IC (1988) Mechanism of the Splice. Textile Research Journal 58(5): 263-268.

5. Bissmann D (1981) La realisation de rattaches de fils sans noeuds par le procédé par épissure. ITB 3/81 Filature, 281-312.

6. Schlafhorst, Schlafhorst / Saurer Group Autoconer 338: 37.

7. Ye Guoming, Li Ying, Chen Ruiqi (1997) A Study on the Splicing Theory of the Air Splicer on the Auto-Coner[J]. Journal of China Textile University 23(2):79-81.

8. KPS Cheng, LI Lam (2000) Physical Properties of Pneumatically Spliced Cotton Ring Spun Yarns. TRJ 70(12): 1053-1057.

9. VK Kothari, AK Sengupta, JK Sensarma (1996) Formation of Neps in AirJet Texturing Using a Central Composite Design. TRJ 66(6): 384-388.

10. Kaushik RCD, Hari PK, Sharma IC (1988) Quantitative Contribution of Splice Elements. Textile Res J 58(6): 343-344.

11. R Hagege, J Brassart et I Raye ( 1986) Premiers résultats relatifs à la morphologie transversale des épissures Etude par analyse d'images, Bull Scient ITF 15(57): 29-47.

12. B Jaouachi, M Ben Hassen, F Sakli (2007) strength of wet spliced denim yarns after sizing using a central composite design. AUTEX Research Journal 7(3).

13. Li Ying, Ye Guoming, Chen Ruiqi (1997) On the Mechanism Design and Analysis of the Air Splicer on Auto-Coner. Journal of China Textile University 23(1): 12-18.

14. Chang D, Li D, Li S, Zhou G, Zhao H (2013) Research on the Impact of Vent Pressure on the Yarn Splicing based on ANSYS. Key Engineering Materials Online 584: 50-53.

15. Das A, Ishtiaque SM, Parida JR (2005) Effect of fiber friction, yarn twist, and splicing air pressure on yarn splicing performance. Fibers and Polymers 6(1): 72-78.

16. Hassen MB,Jaouachi B,Sahnoun M,Sakli F (2008) Mechanical properties and appearance of wet-spliced cotton/elastane yarns. The Journal of The Textile Institute 99(2): 119-123.

17. Jaouachi B, Hassen MB, Sahnoun M, Sakli F (2010) Evaluation of wet pneumatically spliced elastic denim yarns with fuzzy theory. The Journal of The Textile Institute 101(2): 111-119.

18. Webb CJ, Waters GT, Thomas AJ, Liu GP, Thomas EJC (2009) Optimising splicing parameters for splice aesthetics for a continuous filament synthetic yarn. The Journal of The Textile Institute 100(2): 141-151.

19. Webb CJ, Waters GT, Liu GP, Thomas C (2009) The influence of yarn count on the splicing of simple continuous filament synthetic yarns. Textile Research Journal 79(3): 195-204.

20. Das A, Ishtiaque SM, Parmar SK (2012) Study on spliced yarns of different spinning technologies. Part I: tensile characteristics. The Journal of the Textile Institute 103(3): 244-255. 
21. Das A, Ishtiaque SM, Parmar SK (2012) Study on spliced yarns of different spinning technologies. PartII: structural characteristics. The Journal of The Textile Institute 103(3): 256-268.

22. Cheng KPS, Lam HLI (2000) Strength of pneumatic spliced polyester/ cotton ring spun yarns. Textile Research Journal 70(3): 243-246.

23. Cheng KPS, Lam HLI (2000) Physical properties of pneumatically spliced cotton ring spun yarns. Textile Research Journal 70(12): 1053-1057.

24. Cheng KPS, Lam HLI (2003) Evaluating and comparing the physical properties of spliced yarns by regression and neural network techniques. Textile Research Journal 73(2): 161-164.

25. Ünal PG, Özdil N, Taşkın C (2009) The effect of fiber properties on the characteristics of spliced yarns part I: Prediction of spliced yarns tensile properties. Textile Research Journal 80(5): 429-438.

26. Ünal PG, Özdil N, Taşkın C (2010) The effect of fiber properties on the characteristics of spliced yarns: part II: prediction of retained spliced diameter. Textile Research Journal 80(17): 1751-1758.

27. Webb CJ, Waters GT, Thomas AJ, Liu GP, Thomas C (2007) The use of the Taguchi design of experiment method in optimizing splicing conditions for a Nylon 66 yarn. The Journal of the Textile Institute 98(4): 327-336.

28. Moqeet A, Jabbar A, Hussain T, Ali Z, Haq Z U (2013) Influence of splicing parameters on retained splice strength, elongation and appearance of spliced cotton/flax blended yarn. Indian Journal of Fiber \& Textile Research 38(1): 74-80.
29. H Celik, HK Kaynak (2017) Effect of fiber blending ratios of cotton/ polyester yarns on retained splice Diameter. IOP Conf Series: Materials Science and Engineering 254.

30. Kaushik RCD, Hari PK, \& Sharma IC (1987) Textile research journal 57: 670.

31. Lam HL, Cheng KPS (1997) Textile Asia 28: 66.

32. Kaushik RCD, Hari PK, Sharma IC (1988) Textile research journal 58: 263(a) and 343(b).

33. Cheng KPS, Lam HLI (2000) Textile Research Journal 1053(a) and 243(b).

34. Das A, Ishtiaque SM, Nagaraju V (2004) Fibres and Polymers 5: 204.

35. Das A, Ishtiaque SM, Joyoti RP (2005) Fibres and Polymers 6: 72.

36. Webb CJ, Waters GT, Liu GP, Clive (2009) Thomas Textile Research journal 79: 195.

37. Abdul M, Abdul J, Tanveer H, Zulfiqar A, Zahoor UH (2013) Influence of splicing parameters on retained splice strength, elongation and appearance of spliced cotton/flax blended yarn. Indian journal of fibres and textile research 38: 74

\section{(C) This work is licensed under Creative Commons Attribution 4.0 License}

Submission Link:

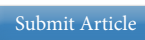

DOI: $10.32474 /$ LTTFD.2018.01.000107

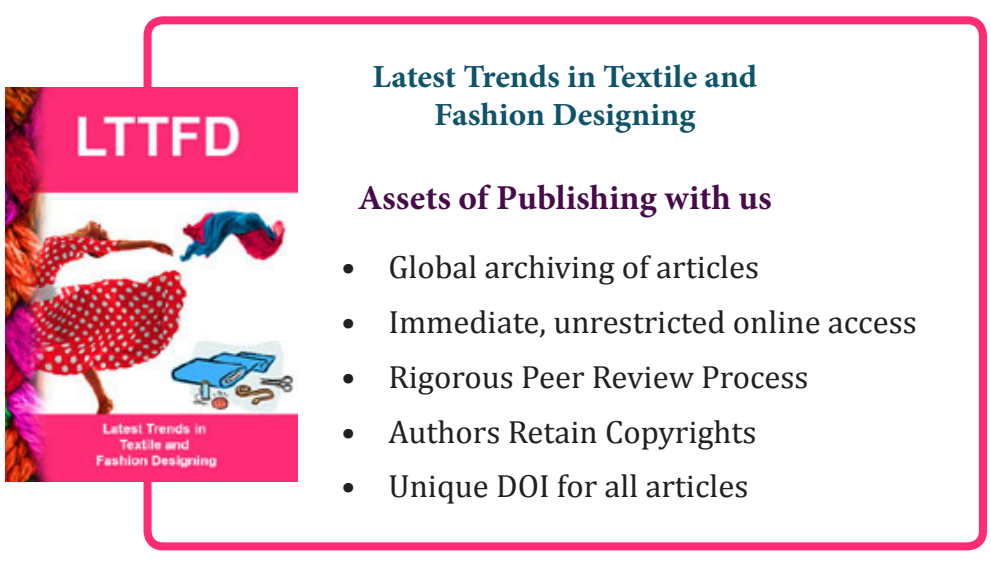

